\title{
Opiniones y respuestas: Resultados de una encuesta de opinión a médicos mexicanos sobre el aborto
}

Diana K. Lara

Lisa Goldman

Michelle Firestone

Follow this and additional works at: https://knowledgecommons.popcouncil.org/departments_sbsr-rh

Part of the Family, Life Course, and Society Commons, Gender and Sexuality Commons, and the International Public Health Commons

How does access to this work benefit you? Let us know!

\section{Recommended Citation}

Lara, Diana K., Lisa Goldman, and Michelle Firestone. 2004. "Opiniones y respuestas: Resultados de una encuesta de opinión a médicos mexicanos sobre el aborto." Mexico City: Population Council. 

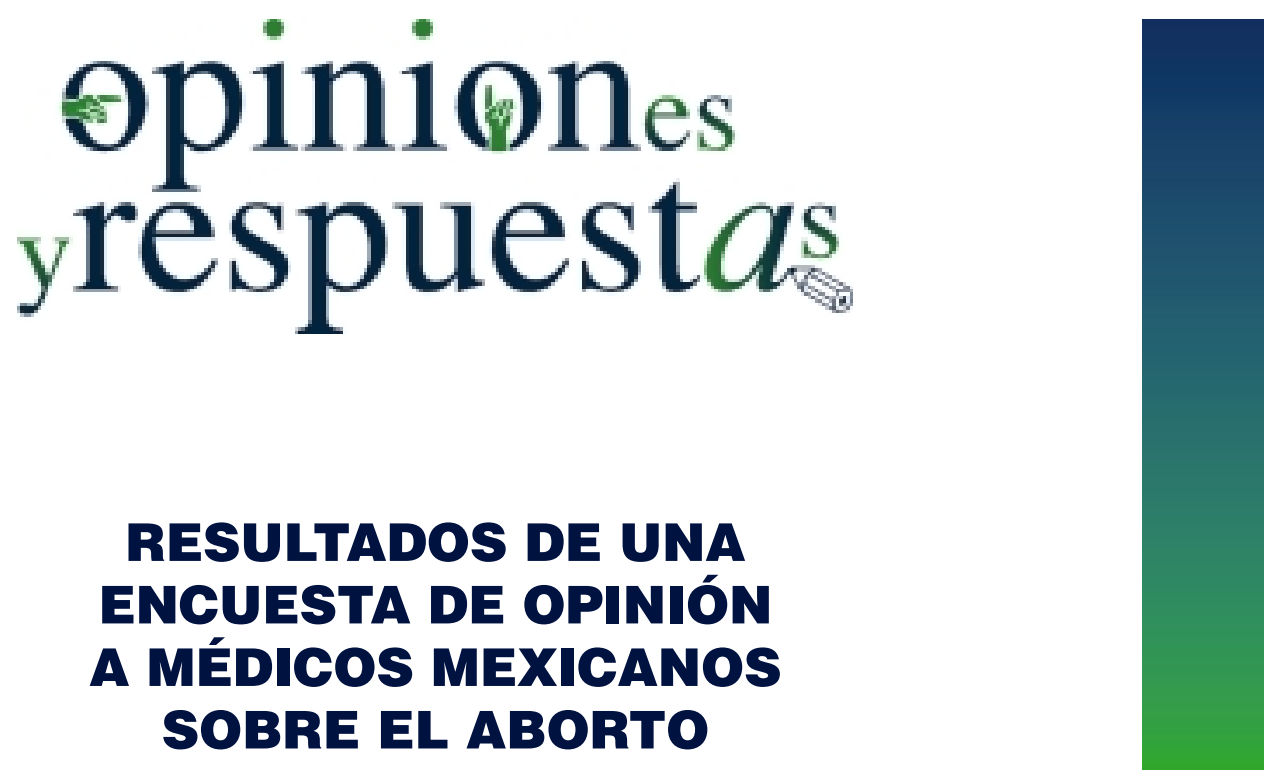

RESULTADOS DE UNA ENCUESTA DE OPINIÓN A MÉDICOS MEXICANOS SOBRE EL ABORTO

12 Population Council 
Esta publicación fue preparada por Diana Lara, Lisa Goldman y Michelle Firestone. La corrección de estilo y el cuidado de la edición estuvieron a cargo de Guillermina Herrera. El diseño fue realizado por Arroyo + Cerda S.C. y la impresión fue responsabilidad de Editiorial Sestante, S.A. de C.V.

La investigación que dio lugar a esta publicación fue realizada por Sandra García y Diana Lara, respectivamente, Directora del Programa Regional de Salud Reproductiva del Population Council, e Investigadora Regional Asociada del mismo programa, Oficina Regional para América Latina y el Caribe, México, D.F.

Primera Edición, 2004.

(c) D.R. Population Council

Correo-e: biblioteca@popcouncil.org.mx

\section{Population Council}

El Population Council es una organización internacional, no lucrativa y no gubernamental, que busca mejorar el bienestar y la salud reproductiva de las generaciones presentes y futuras en el mundo y ayudar a alcanzar un equilibrio entre la población y los recursos. El Council realiza investigación biomédica, en ciencias sociales y salud pública, y ayuda a fortalecer la capacidad de investigación en países en vías de desarrollo. El Council fue fundado en 1952 y está dirigido por una junta directiva multinacional. Su oficina matriz en Nueva York apoya una red global de oficinas regionales y nacionales.

Population Council

One Dag Hammarskjold Plaza

New York, NY 10017 USA

http://www.popcouncil.org

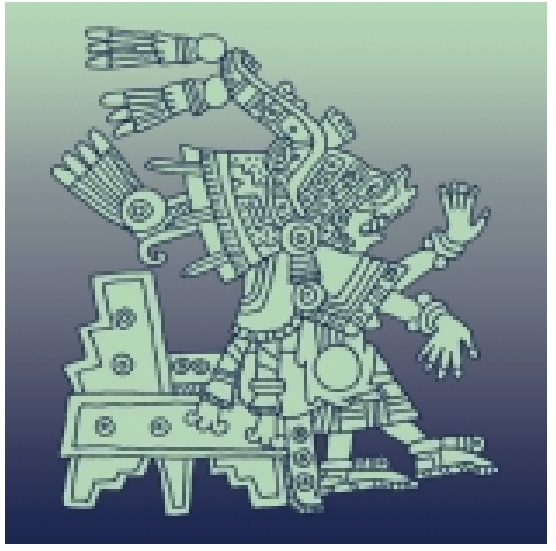

Xochiquetzal, arquetipo de las actividades artísticas de las mujeres. Borgia, 9.

Las ilustraciones y pies de figura que complementan esta publicación fueron tomados del capítulo 5 del libro 'Las diosas en los códices del Grupo Borgia' de María de los Ángeles Ojeda Díaz: www.arts-history.mx/diosas.cap51.html\#xochi

\section{AGRADECIMIENTOS}

Las instituciones que colaboraron en la realización del estudio y en la preparación de esta publicación son: Secretaría de Salud de México, Grupo Investigación de Mercado y Asesoría (IDM), Universidad Autónoma Metropolitana, Unidad Xochimilco, Secretaría de Salud del Gobierno del Distrito Federal, Instituto Mexicano del Seguro Social, Academia Nacional de Medicina e Ipas México.

Agradecemos a Sandra García, Daniel Grossman y Ana Langer su asesoría en este proyecto. Del mismo modo, hacemos patente nuestro agradecimiento a las siguientes personas que colaboraron en diferentes aspectos del mismo: Marilú Alarcón, Tess Aldrich, Javier Arellano, Davida Becker, Deborah Billings, Leticia de la Cruz, Claudia Díaz, Charlotte Ellertson, Alejandra Gasca, Stanley Henshaw, Anjarath Higuera, Martha Juárez, Doroteo Mendoza, Alicia Mesa, Claudia Moreno, Rafael Lozano, Maricarmen Meza, Francisco Paz, Patricio Sanhueza, Raffaela Schiavon, Irvin Sivin, Julio Sotelo, Javier Suárez, Salme Tamus, David Trejo y Raúl Wong. 


\section{- Introducción}

América Latina y el Caribe es la región con la tasa de abortos inseguros más elevada en el mundo: en ella ocurren cada año 3.7 millones de abortos, estimándose una tasa de 26 por 1,000 mujeres en edad reproductiva. ${ }^{1}$ Los abortos inseguros son la causa de $25 \%$ de todas las muertes maternas que suceden en América Latina y el Caribe, de alrededor de $12 \%$ de las que ocurren en África, y de $10 \%$ de las que suceden en Asia. ${ }^{2}$ Se estima que en México la mortalidad asociada con el aborto inseguro es la tercera o cuarta causa de las muertes maternas; ; ${ }^{3,4}$ también en el ámbito mundial es uno de los problemas de salud más graves, complejos y prevenibles que enfrentan la salud pública y la comunidad médica actualmente., ${ }^{2,5}$

En México es incuestionable el papel crucial que los médicos juegan en la oferta de servicios seguros de aborto legal y en la atención a las mujeres con abortos en evolución, espontáneos o inducidos, y abortos complicados. El Instituto Alan Guttmacher estimó que en 1990 se practicaron en México más de 500,000 abortos ilegales (una tasa similar a la mitad de los abortos legales que ocurren en Estados Unidos), lo que llevó a la hospitalización por complicaciones a más de 100,000 mujeres. ${ }^{6,7}$

Diversos estudios han explorado la opinión pública sobre el aborto de la población en general en México; ${ }^{8-11}$ otros han explorado en específico la opinión del personal de salud sobre este tema; ${ }^{12-17}$ sin embargo, ningún estudio dirigido a personal de salud, y específicamente a médicos, se había realizado con una muestra representativa a nivel nacional. Una brecha de ese tipo en la literatura científica justificó la realización, entre julio y agosto de 2002 , de una encuesta nacional ${ }^{18}$ en la cual recopilamos información sobre los conocimientos y las opiniones de médicos gíneco-obstetras, médicos familiares y médicos generales, sobre los aspectos legales y médicos del aborto en México. También recopilamos información sobre la experiencia de los médicos en la práctica de abortos legales y el manejo de abortos incompletos y sus complicaciones, así como sus necesidades en cuanto a información y capacitación técnica.

En este folleto se brinda información científica y actualizada de los temas de interés mencionados con más frecuencia por los médicos que participaron en la encuesta.

Para información adicional sobre aborto —investigación, aspectos éticos, leyes y otros aspectos-, consulte la lista de recursos en internet que se encuentra al final de la presente publicación. 


\section{Metodología}

Se aplicó un cuestionario auto-administrado a una muestra de médicos gínecoobstetras (MGO), médicos familiares (MF) y médicos generales (MG) que laboran en clínicas y hospitales de la Secretaría de Salud (SSA) y del sector privado localizadas en zonas urbanas de todo el país. En el estudio participaron 288 unidades de servicios de salud en las cuales se entrevistaron a 1,206 médicos. La tasa de no respuesta fue de $24 \%$.

Este cuestionario auto-administrado fue anónimo y confidencial y comprendió preguntas sobre conocimientos, actitudes y prácticas en relación con el aborto legal e ilegal. También se indagó sobre la disponibilidad de los médicos para participar en servicios de aborto legal, aceptación de la introducción del aborto con medicamentos y temas de capacitación y actualización. La recolección de la información se llevó a cabo del 2 de julio al 16 de agosto de 2002.

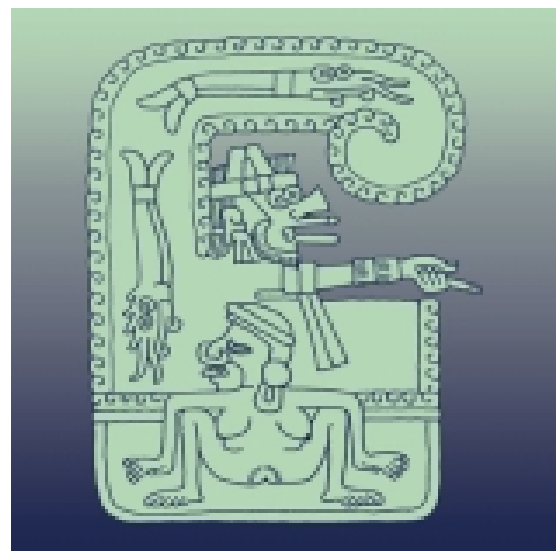

Tlazolteotl, en posición de parto. Laud, 40.

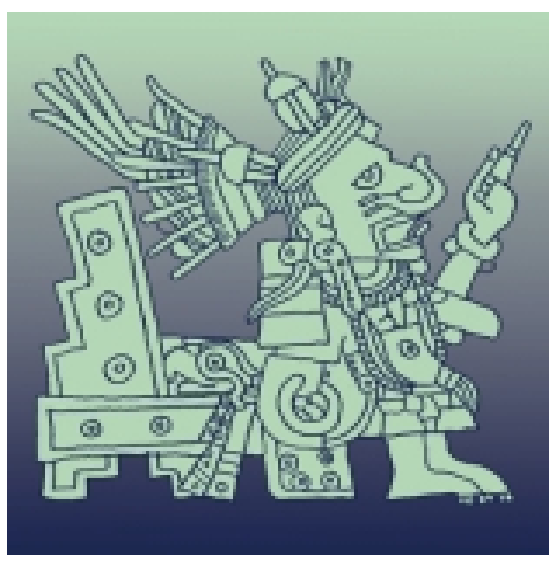

Tlazolteotl, arquetipo de la Madre, de la mujer madura. Borgia, 12 


\section{- Perfil de los participantes}

La mayoría de los participantes del estudio eran hombres (70\%). Sesenta y nueve por ciento de los participantes tenían entre 34 y 53 años de edad y $85 \%$ se identificó a sí mismo como católico. Casi la mitad de las y los participantes eran médicos generales (54\%), seguidos por gíneco-obstetras (25\%) y médicos familiares $(21 \%)$. Una proporción importante había egresado de universidades públicas: $32 \%$ de la Universidad Nacional Autónoma de México (UNAM) y 57\% de otras universidades públicas. Trabajaba exclusivamente en el sector público $33 \%$ de los médicos, mientras $27 \%$ se desempeñaba tanto en el sector público como el privado. En su mayoría, los médicos que laboraban en el sector público lo hacían en el servicio de consulta externa (79\%), $27 \%$ en el servicio de urgencias y $22 \%$ en hospitalización. Pocos participantes eran jefes de departamento (7\%), mientras que casi tres cuartas partes $(72 \%)$ eran médicos adscritos.

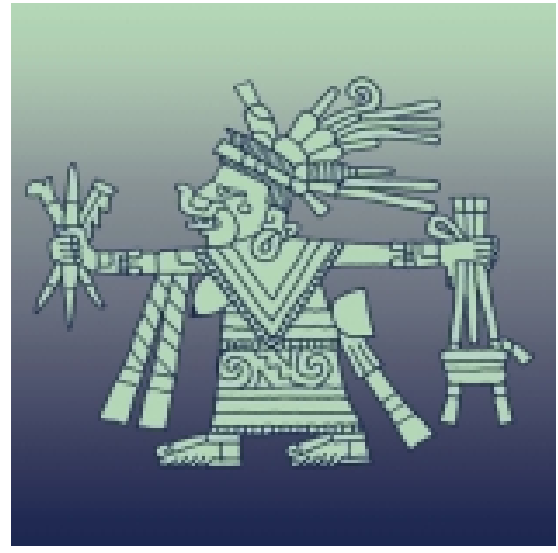

Tlazolteotl, arquetipo de la mujer madura. Laud, 42.

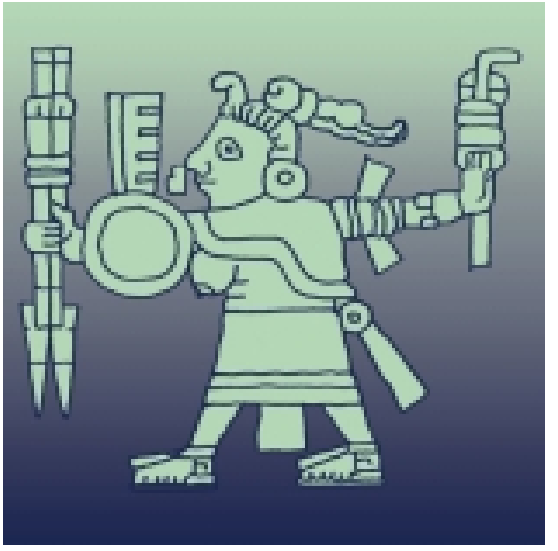

Tlazolteotl, arquetipo de la mujer guerrera. Laud, 20 


\section{- Temas de interés}

Aproximadamente la mitad o más de las/os médicos señalaron que les gustaría recibir mayor información sobre los temas que se presentan en el cuadro 1.

- Cuadro 1. Temas de interés mencionados por los participantes

Tema

$n=1,206$

\begin{tabular}{ll} 
Aspectos legales & $\%$ \\
\hline Aspectos éticos & 81 \\
\hline Uso de medicamentos para aborto & 65 \\
(prostaglandinas, mifepristona, metotrexate) & 56 \\
\hline Técnicas quirúrgicas y farmacológicas para aborto & \\
\hline Aspiración manual endouterina (AMEU) & 55 \\
\hline Legrado uterino instrumental (LUI) & 52
\end{tabular}

Otros temas de capacitación que mencionaron menos de la mitad de los médicos abarcaban: apoyos diagnósticos de gabinete (ultrasonido), interacción médicopaciente, aspectos epidemiológicos del aborto, uso de anestesia en el aborto, diagnóstico, clasificación clínica y definición.

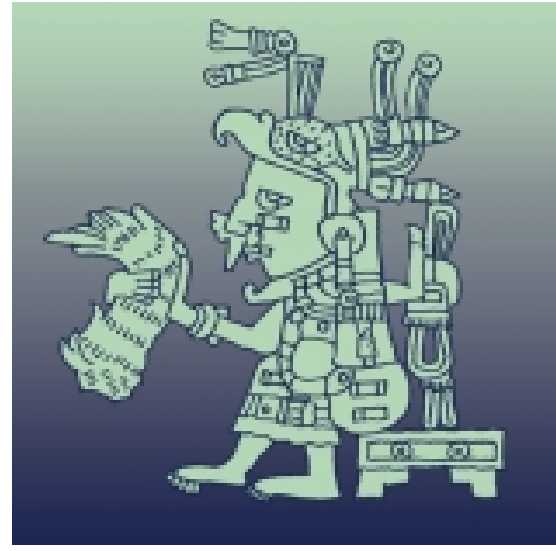

Xochiquetzal, arquetipo de la mujer joven. Borgia, 57.

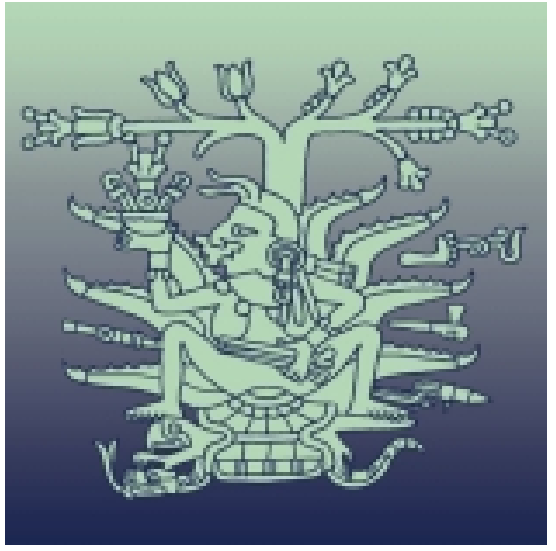

Mayahuel, como Ayopechtli, "la que está sobre la tortuga" protectora de los vientres maduros que se hacen vida. Laud, 9. 


\section{- Aspectos legales}

Aunque un alto porcentaje de médicos sabía que el aborto es legal bajo algunas circunstancias, algunos desconocían en qué situaciones específicas estaba permitido en la localidad estatal en la cual laboraban.

\section{- Cuadro 2. Conocimiento del marco legal del aborto en el estado en donde se desempeñan}

\begin{tabular}{lc} 
Conocimiento & $\mathrm{n}=1,206$ \\
$\%$ & 83 \\
El aborto es legal en algunas circunstancias & 16 \\
\hline El aborto no es legal en ninguna circunstancia & 1
\end{tabular}

La figura 1 ofrece información de las leyes estatales acerca del aborto permitido por violación, peligro de muerte, malformaciones congénitas y grave daño a la salud.

El código penal de las distintas entidades estatales establece las causales bajo las cuales el aborto es legal en cada una de ellas; sin embargo, en muchos estados aún no se ha definido en el código de procedimientos el mecanismo administrativo para obtener la intervención, y tampoco existen lineamientos médicos dictados por las Secretarías de Salud de los estados que establezcan procedimientos al interior de los hospitales a fin de garantizar la oferta de servicios de aborto legal.

El Distrito Federal se encuentra a la vanguardia de los estados en el establecimiento de procedimientos administrativos y médicos para la oferta de servicios de aborto legal. Aquí, la Secretaría de Salud local emitió, en abril de 2002, los "Lineamientos generales de organización y operación de los servicios de salud relacionados con la interrupción del embarazo en el Distrito Federal", ${ }^{19}$ estipulando que los médicos deben practicar el aborto bajo las circunstancias contempladas por la ley: si el embarazo es menor de 20 semanas, en un plazo de diez días naturales a partir de la primera consulta en la unidad médica. Establece, además, que en caso de que el médico se abstenga de practicar el aborto por razones de conciencia, debe referir a la paciente con otro médico o a otra institución para que se le brinde el servicio. 
- Figura 1. Información sobre causales bajo las cuales el aborto es legal, por estados del país

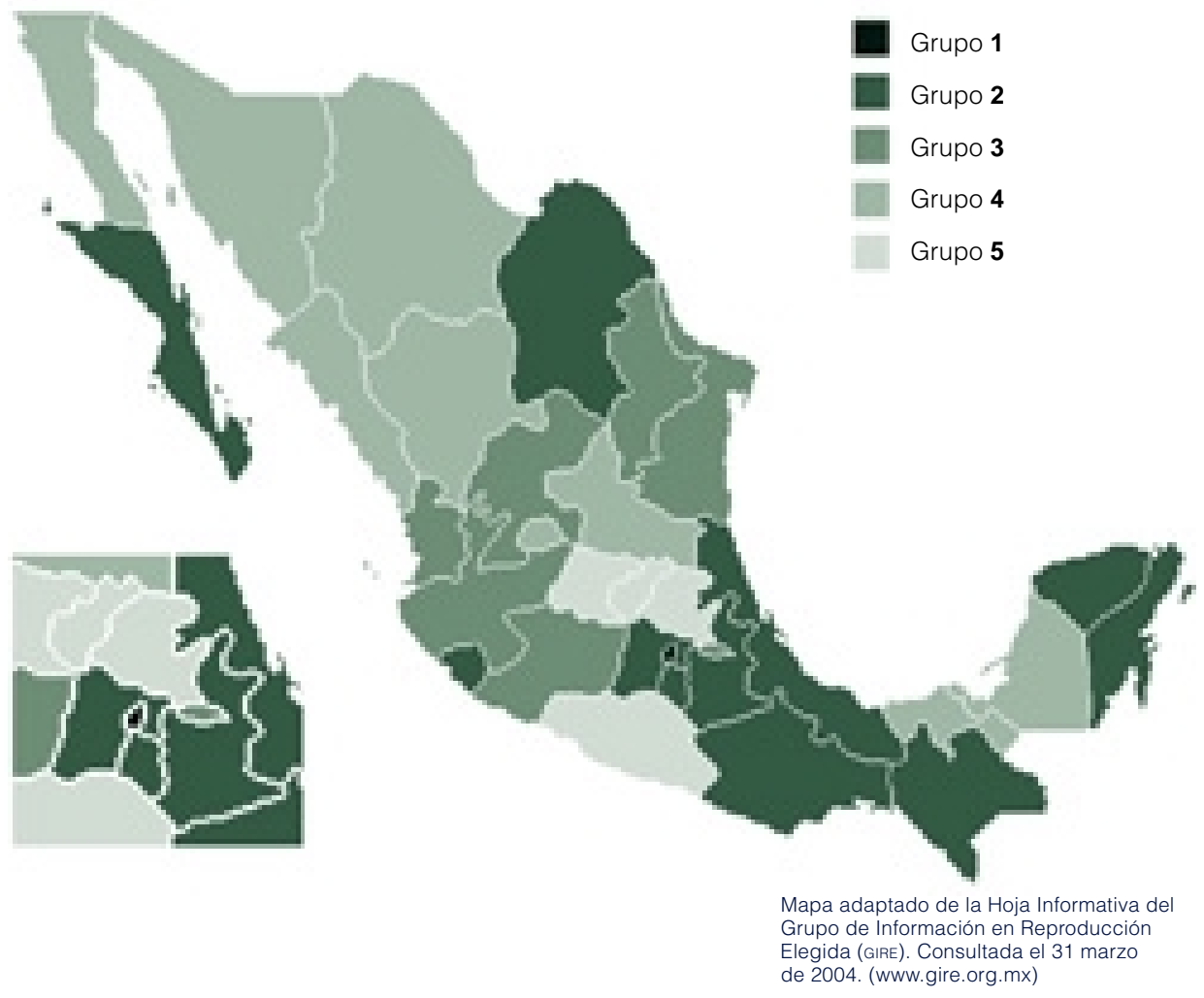

Grupo Circunstancias bajo las cuales el aborto es legal

$1^{\text {a }} \quad$ Violación, peligro de muerte, malformaciones congénitas, grave daño a la salud

2 Violación, peligro de muerte, malformaciones congénitas

3 Violación, peligro de muerte, grave daño a la salud

$4 \quad$ Violación, peligro de muerte

$5^{\text {b }} \quad$ Violación

a En agosto de 2000 se modificó la causal de peligro de muerte y, en su lugar, se agregó grave daño a la salud, que es una circunstancia más amplia.

En Guerrero, el aborto está permitido, además del caso de violación, en situaciones de malformaciones congénitas. Mientras tanto, en Hidalgo, se permite en caso de grave daño a la salud de la mujer, causal que incluye los casos en los que la mujer esta en peligro de muerte. 


\section{- Técnicas quirúrgicas}

En la encuesta se indagó sobre el conocimiento de técnicas quirúrgicas y medicamentos utilizados:

a) para inducir abortos legales cuando el cérvix se encuentra cerrado, y

b) para evacuar el útero cuando el cérvix se encuentra dilatado (abortos en evolución e incompletos).

La técnica quirúrgica más conocida por los médicos fue el legrado uterino instrumental (LUI), tanto para evacuar la cavidad uterina (91\%) como para inducir un aborto legal (72\%). Menos de la mitad de los médicos mencionaron la aspiración manual endouterina (AMEU) para ambas indicaciones. El conocimiento de la AMEU fue mucho menor en el grupo de MF y MG, comparado con los MGO, tanto en la atención de abortos incompletos como en la inducción de abortos legales (ver cuadro 3$)$.

\section{- Cuadro 3. Conocimiento de técnicas quirúrgicas para atender a mujeres que presentan abortos}

\section{Técnicas quirúrgicas}

Evacuación del útero (cérvix dilatado)

- Legrado uterino instrumental (LUI)

- $\quad$ Aspiración manual endouterina (AMEU)

Inducción del aborto (cérvix cerrado)

- $\quad$ Legrado uterino instrumental (LUI)

- $\quad$ Aspiración manual endouterina (AMEU)

\begin{tabular}{c|c|c|c} 
& \multicolumn{3}{|c}{ Especialidad } \\
\cline { 2 - 4 } $\begin{array}{c}\mathrm{n}=1,206 \\
\%\end{array}$ & $\begin{array}{c}\text { MGO } \\
\mathrm{n}=317 \\
\%\end{array}$ & $\begin{array}{c}\text { MF } \\
\mathrm{n}=251 \\
\%\end{array}$ & $\begin{array}{c}\text { MG } \\
\mathrm{n}=638\end{array}$ \\
\hline 91 & 93 & 91 & 91 \\
\hline 49 & 73 & 38 & 42 \\
\hline 72 & 77 & 68 & 73 \\
\hline 48 & 60 & 45 & 43 \\
\hline
\end{tabular}


En la literatura científica se ha demostrado ampliamente que la aspiración, ya sea manual o eléctrica, es una técnica eficaz y más segura y barata que el LUI. ${ }^{20,21}$ Una revisión de 19 estudios clínicos mostró que la aspiración tiene una efectividad superior a $98 \%$ en la resolución de abortos incompletos. Mientras tanto, en 46 investigaciones realizadas en 24 países se ha demostrado que la aspiración tiene tasas de efectividad para el aborto inducido que varían de 87 a 100\%. En una revisión de 12 estudios en los que se usó la AMEU en más de 200,000 mujeres para practicarles abortos inducidos se reportaron tasas de efectividad de 95 a $100 \% .^{22}$

En los países desarrollados, la técnica de aspiración (manual y eléctrica) se utiliza ampliamente desde hace varias décadas. En Estados Unidos, 97\% de los abortos inducidos son atendidos con esta técnica; ${ }^{23}$ sin embargo, en los países en desarrollo sigue predominando el uso del LUI sobre la AMEU. Por ejemplo, en un estudio realizado en Egipto, en el cual se hizo una revisión de las técnicas quirúrgicas usadas en 569 hospitales públicos para atender a pacientes con abortos en evolución, incompletos y diferidos, se encontró que en $98 \%$ de los procedimientos se realizó un LUI. ${ }^{24}$

En cuanto a seguridad, estudios comparativos muestran que las tasas de complicaciones son mucho menores con la aspiración que con el LUI. En una investigación realizada en Estados Unidos, en la cual se evaluaron 1,677 AMEU en embarazos de 7 a 12 semanas, se encontró que sólo en 1.25 de 100 procedimientos se presentaron complicaciones menores. ${ }^{25}$

Los datos tomados de un estudio en seis hospitales del Instituto Mexicano del Seguro Social (IMSS) en el Distrito Federal ${ }^{26}$ indican que el uso de la AMEU y el LUI son comparables en términos de seguridad. Los médicos registraron pocas complicaciones obstétricas o sistémicas durante el procedimiento y post-procedimiento (ver cuadro 4).

Johnson y colaboradores ${ }^{27}$ realizaron un estudio en hospitales de Kenia y la ciudad de México para valorar las diferencias de costo entre la AMEU y el LUI. Se reportó entonces que en el hospital de México el costo promedio de un LUI era el equivalente a $\$ 143.25$ dólares, mientras que el de la AMEU era el equivalente a \$65.73 dólares, lo que representa una reducción en costos para el hospital de más de la mitad. Otro estudio realizado en un hospital público de la ciudad de Oaxaca, en México, ${ }^{28}$ reportó que el costo de la AMEU era 32\% menor ( $\$ 180.22$ dólares) comparado con el costo del LUI (\$264.47 dólares). 
- Cuadro 4. Seguridad de las técnicas AMEU y LUI

\begin{tabular}{|c|c|c|c|}
\hline \multirow{2}{*}{\multicolumn{2}{|c|}{ Complicaciones }} & \multicolumn{2}{|c|}{ Seguridad } \\
\hline & & $\begin{array}{l}\text { AMEU } \\
n=232\end{array}$ & $\begin{array}{c}\text { LUI } \\
n=269\end{array}$ \\
\hline & nte el procedimiento & $\%$ & $\%$ \\
\hline$\bullet$ & Obstétricas a & 0.0 & 0.7 \\
\hline$\bullet$ & Sistémicas b & 3.4 & 2.6 \\
\hline \multicolumn{4}{|c|}{ Post-procedimiento } \\
\hline & Obstétricas ${ }^{\mathrm{c}}$ & 0.4 & 0.4 \\
\hline & Sistémicas $^{d}$ & 0.0 & 0.0 \\
\hline
\end{tabular}

\footnotetext{
a Laceración cervical, ingreso de aire en la cavidad abdominal.

b Convulsiones, reacción alérgica, broncoaspiración, reacción vagal, somnolencia, hipertensión arterial, estimulación cortical.

Sangrado intrauterino persistente, sangrado cervical.

Shock hipovolémico, depresión cortical persistente, depresión respiratoria persistente, hipertensión arterial persistente.

Fuente: Fuentes J, Billings D. A comparison of three models of postabortion care in Mexico. Final report. Population Council. New York, 1998.
}

Otra de las ventajas que ofrece la AMEU es que puede realizarse bajo anestesia local en vez de anestesia general, la cual se utiliza generalmente en los LUI. Usualmente se realiza un bloqueo paracervical para reducir el dolor derivado de la dilatación y manipulación del cérvix. ${ }^{29}$ Este tipo de anestesia disminuye los riesgos durante el procedimiento y es menos costosa que la anestesia general. ${ }^{30}$ Además, la anestesia local puede ofrecerse en servicios de salud de primer nivel facilitando la descentralización del servicio y mejorando el acceso a éste de las mujeres que viven en áreas lejanas a hospitales de segundo y tercer nivel.

La aspiración es la técnica más utilizada para la atención de abortos legales en países desarrollados y existe un fuerte respaldo científico sobre su efectividad, seguridad, bajo costo y fácil uso. ${ }^{31,32}$

En México, desde hace algunos años el uso de la AMEU se está incluyendo como tema de enseñanza en los programas de las facultades de medicina más importantes del país ${ }^{33}$ y se ha incrementado el uso y la capacitación correspondiente en los hospitales públicos. ${ }^{34}$ 


\section{- Técnicas farmacológicas}

Por otra parte, más de la mitad de las y los entrevistados conocían el uso del medicamento misoprostol para la inducción de un aborto (61\%), mientras que una proporción menor conocía los regímenes combinados, científicamente probados y utilizados con frecuencia en países desarrollados, como mifepristona más misoprostol (43\%), y metotrexate más misoprostol (34\%) (ver cuadro 5). La mitad de los entrevistados sabía del uso del misoprostol para la atención de abortos incompletos. El nivel de conocimiento sobre misoprostol para la atención de abortos incompletos y para inducir abortos fue similar en los MGO, MF y MG.

A pesar de que existe un bajo conocimiento sobre los regímenes de aborto médico, los médicos expresaron interés en recibir cursos de entrenamiento y capacitación en este tema; $56 \%$ dijo que quería aprender más sobre el aborto legal con medicamentos.

\section{- Cuadro 5. Conocimiento de medicamentos para la atención} de abortos

Técnicas quirúrgicas

Evacuación del útero (cérvix dilatado)

- $\quad$ Misoprostol $\left(\right.$ Cytotec $\left.^{\circledR}\right)$ solo u otras prostaglandinas solas

Inducción del aborto (cérvix cerrrado)

- $\quad$ Misoprostol $\left(\right.$ Cytotec $\left.^{\circledR}\right)$ solo $\mathrm{u}$ otras prostaglandinas solas

- $\quad$ Mifepristona (RU-486) + misoprostol (Cytotec $^{\circledR}$ )

- $\quad$ Metotrexate $\left(\right.$ Ledertrexate $\left.^{\circledR}\right)+$ misoprostol (Cytotec $^{\circledR}$ )

\begin{tabular}{cc|c|c} 
& \multicolumn{3}{|c}{ Especialidad } \\
\cline { 2 - 4 } $\begin{array}{c}\text { MGO } 1,206 \\
\%\end{array}$ & $\begin{array}{c}\text { MF }=406 \\
\%\end{array}$ & $\begin{array}{c}\text { MF }=426 \\
\%\end{array}$ & $\begin{array}{c}\text { MG } \\
\%\end{array}$ \\
49 & 50 & 49 & 50 \\
\hline 61 & 69 & 51 & 57 \\
\hline 43 & 53 & 37 & 36 \\
\hline 34 & 37 & 31 & 27 \\
& & &
\end{tabular}


Además de la AMEU y el LUI, es posible también utilizar medicamentos para atender de forma efectiva y segura los abortos en evolución e inducidos. El régimen más estudiado, efectivo y seguro para la inducción de un aborto es la combinación de mifepristona más misoprostol. Sin embargo, dado que en México no se encuentra aún disponible la mifepristona, es posible utilizar otro régimen que ha sido ampliamente estudiado también: la combinación de metotrexato con misoprostol.

La mifepristona, llamada antes $R U-486$, fue desarrollada en Francia y aprobada en ese país desde 1988. ${ }^{35}$ Esta sustancia bloquea los receptores de progesterona inhibiendo la acción de la hormona necesaria para la implantación y mantenimiento de la placenta. ${ }^{36} \mathrm{El}$ medicamento se ha aprobado en al menos 18 países en todo el mundo. ${ }^{37}$

El misoprostol, mejor conocido por su marca comercial Cytotec ${ }^{\circledR}$ es una prostaglandina $\mathrm{E}_{1}$ análoga cuya indicación principal es la prevención y tratamiento de úlceras gástricas. Este medicamento se encuentra disponible en más de 80 países en todo el mundo. ${ }^{38}$ En México, el producto lo vende el laboratorio Pfizer y su costo al público oscila entre $\$ 612$ y $\$ 875$ pesos; la presentación es de 28 tabletas de 200 microgramos.

El medicamento se puede administrar por vía oral o vaginal, aunque existe evidencia que su eficacia es mayor administrada por vía vaginal. ${ }^{39} \mathrm{El}$ misoprostol actúa en los receptores de prostaglandinas produciendo contracciones uterinas; además, suaviza y dilata el cérvix y tiene la ventaja de que se puede almacenar a temperatura ambiente. ${ }^{39}$

El metotrexate se encuentra disponible en México bajo la marca comercial Ledertrexate ${ }^{\circledR}$. Se ha usado desde hace más de 40 años para el tratamiento de enfermedades neo-plásicas, artritis reumatoide y psoriasis. Esta droga bloquea la enzima dihidrofolato reductasa, la cual esta involucrada en la producción de timidina durante la síntesis del ácido desoxiribonucleico (ADN). El metotrexate actúa sobre el citotrofoblasto más que sobre el embrión; de allí su utilidad en el tratamiento del embarazo extrauterino que comúnmente es anembriónico. Lo que el metotrexate hace básicamente es interrumpir el proceso de implantación. ${ }^{40}$

En el siguiente cuadro se muestra la dosis, vía de administración, edad gestacional límite y efectividad de los principales regímenes del aborto médico. 
- Cuadro 6. Regímenes utilizados para el aborto médico

\begin{tabular}{|c|c|c|c|}
\hline Régimen a & Dosis y vía de administración & Edad gestacional límite & Efectividad \\
\hline $\begin{array}{l}\text { Mifepristona } \\
+ \text { misoprostol }^{\mathrm{b}} \\
\text { (régimen aprobado } \\
\text { por la FDA) }\end{array}$ & $\begin{array}{l}\text { Mifepristona } 600 \text { mg. vía } \\
\text { oral + misoprostol } 400 \text { mcg. } \\
\text { (48 horas después de la } \text { mifepristona) }{ }^{d}\end{array}$ & $\begin{array}{l}49 \text { días o menos después } \\
\text { de la última menstruación }\end{array}$ & 92 a $97 \%$ \\
\hline $\begin{array}{l}\text { Mifepristona } \\
+ \text { misoprostol }^{\mathrm{b}} \\
\text { (régimen basado } \\
\text { en resultados de } \\
\text { estudios clínicos) }\end{array}$ & $\begin{array}{l}\text { Mifepristona } 200 \text { mg. vía } \\
\text { oral + misoprostol } 800 \text { mcg. } \\
\text { vía vaginal ( } 24 \text { a } 72 \text { horas } \\
\text { después del mifepristone) }{ }^{d}\end{array}$ & $\begin{array}{l}63 \text { días o menos después } \\
\text { de la última menstruación }\end{array}$ & $97 \%$ \\
\hline $\begin{array}{l}\text { Metotrexate } \\
+ \text { misoprostol }^{\mathrm{c}}\end{array}$ & $\begin{array}{l}\text { Metotrexate } 50 \text { mg/m², } \\
\text { intramuscular + misoprostol } \\
800 \text { mcg. vía vaginal } \\
\text { (3 a } 7 \text { días después del } \\
\text { metotrexate) }{ }^{d}\end{array}$ & $\begin{array}{l}49 \text { días o menos después } \\
\text { de la última menstruación }\end{array}$ & 90 a $96 \%$ \\
\hline
\end{tabular}

En los pocos casos en los cuales falle alguno de estos regímenes de aborto médico, es necesario llevar a cabo una evacuación quirúrgica del útero. El uso de misoprostol, mifepristona y metotrexate durante el primer trimestre es potencialmente teratogénico. El uso del misoprostol se ha asociado con el síndrome de Möbius, y el metotrexate con anomalías digitales y craneofaciales. Ver Goldberg y cols. $2001^{41}$

Stewart y cols., $2001^{35}$

Creinin y cols., $1995^{42}$

Si una semana después de haber finalizado el tratamiento se confirma un aborto incompleto, se puede repetir una dosis adicional de 800 mcg de misoprostol vía vaginal y dar una semana más de espera. Espinoza y cols., $2003^{37}$

El régimen de mifepristona más misoprostol, aprobado por la USA Food and Drug Administration (FDA), tiene una efectividad muy alta; sin embargo, su costo es elevado ya que la mifepristona es un medicamento caro y cuyo costo varía de un país a otro (en Estados Unidos el costo de una tableta de $200 \mathrm{mg}$. es de $\$ 90$ dólares mientras que en China e India es alrededor de $\$ 6$ dólares). ${ }^{37}$ Por tal razón, se han realizado estudios clínicos para probar la efectividad de un régimen con una dosis menor de mifepristona. Derivado de estos estudios, existe otro régimen con alta efectividad y muy utilizado en diferentes países en el cual se administran $200 \mathrm{mg}$. de mifepristona seguidos de $400 \mathrm{mcg}$. de misoprostol por vía vaginal uno o dos días después de la administración de la mifepristona. ${ }^{37}$ 
Los efectos secundarios más frecuentes derivados del uso del régimen de mifepristona y misoprostol son: naúseas (36 a 67\%), dolor de cabeza (13 a 32\%), vómito (13 a 34\%), diarrea (8 a 23\%), mareos (12 a 37\%), fiebre o escalofríos (4 a $37 \%) .{ }^{35}$ También se presenta dolor abdominal tipo cólico y sangrado vaginal como parte inherente del proceso de aborto. ${ }^{37}$

El uso del régimen de metotrexate más misoprostol es una alternativa de tratamiento en aquellos países en dónde la mifepristona no está disponible. El proceso de aborto es más lento cuando se utiliza este régimen en comparación con el régimen de mifepristona. ${ }^{37}$ Además del uso para la inducción de abortos legales, la sustancia es ampliamente utilizada para el tratamiento de embarazos ectópicos. ${ }^{43}$

El misoprostol solo se ha utilizado en diferentes procedimientos obstétricos, tales como inducción de un aborto (aborto médico temprano), dilatación cervical previa a un legrado quirúrgico, aborto incompleto, huevo muerto retenido, inducción del trabajo de parto en caso de muerte fetal intrauterina, inducción del trabajo de parto y hemorragia postparto. ${ }^{41}$

En el caso específico del uso del misoprostol solo en aborto médico, algunos estudios muestran una efectividad que oscila entre 19 y $97 \% .{ }^{39}$ Los estudios más recientes reportan una efectividad alrededor de $80 \% .^{44-47}$ Resultados tan diversos como éstos se deben a diferencias en el diseño de los estudios, las dosis, número e intervalo entre ellas, vía de administración, poblaciones, semanas de embarazo cuando se aplicó el régimen y parámetros de definición de un aborto exitoso. Es necesario realizar más investigación en este campo para definir una dosis y ruta de administración adecuada para el uso del misoprostol como régimen de aborto médico.

En cuanto al uso del misoprostol solo para la atención de abortos incompletos, un estudio clínico controlado reportó una efectividad de $61 \%$ utilizando una dosis de $800 \mathrm{mcg}$. de misoprostol por vía vaginal y de $64 \%$ administrando una dosis similar por vía oral. ${ }^{48}$ 


\section{- Opinión}

Cuando se indagó la opinión de los médicos acerca de la calidad legal del aborto en circunstancias específicas, se observó una tendencia a aceptar la práctica del aborto bajo las condiciones que ya están incluidas en el Código Penal, y mayor resistencia a aceptarla por problemas económicos o por falla de un método anticonceptivo, cuando la mujer es madre soltera, menor de edad o por decisión propia (cuadro 7).

\section{- Cuadro 7. Circunstancias bajo las cuales las y los médicos opinan que el aborto debería ser legal y cuándo estarían dispuestos/as a practicar un aborto}

Circunstancias

El embarazo es resultado de una violación

La mujer embarazada corre peligro de muerte

La mujer embarazada corre peligro de

afectación grave a su salud

El producto puede tener alteraciones

genéticas o congénitas graves

El embarazo es resultado de una inseminación

artificial no deseada ni consentida

\begin{tabular}{lc|c}
\hline Falta de recursos económicos & 13 & 7 \\
\hline La mujer es madre soltera & 8 & 4 \\
\hline La mujer es menor de edad (menos de 18 años) & 13 & 8 \\
\hline Falla de un método anticonceptivo & 15 & 10 \\
\hline La mujer así lo decide & 20 & 11
\end{tabular}

a Respuesta a la pregunta: "¿Qué tan de acuerdo o en desacuerdo está usted con que el aborto sea legal o no en las siguientes circunstancias?"

b Respuesta a la pregunta: "En algunas de las siguientes circunstancias el aborto es legal y en otras no lo es. ¿Bajo cuáles de las siguientes circunstancias estaría usted dispuesto/a a realizar un aborto si una paciente se lo solicita y usted tuviera el suficiente entrenamiento para realizarlo?" 
Además de indagar sobre la opinión en cuanto a la legalidad del aborto, preguntamos sobre la disponibilidad de practicar abortos bajo esas mismas circunstancias si se tuvieran los conocimientos necesarios. Una proporción considerable de las y los entrevistados manifestaron una actitud favorable hacia la legalidad del aborto bajo ciertas circunstancias; sin embargo, un porcentaje menor manifestó que estaría dispuesto a practicar un aborto bajo esas mismas circunstancias. En el cuadro 7 se puede observar que $86 \%$ de los entrevistados de ambos sexos reportaron estar de acuerdo con la legalidad del aborto en caso de violación; no obstante, sólo $71 \%$ manifestó que practicaría un aborto en tal circunstancia.

Del mismo modo, una alta proporción (84\%) de las y los médicos que participaron en esta encuesta señalaron que los hospitales públicos deberían ofrecer servicios de aborto en los casos permitidos por la ley, mientras poco más de la mitad dijo estar dispuesto/a a realizar intervenciones legales. Sólo 11\% dijo que en el transcurso de su vida profesional había hecho alguna vez un aborto legal.

\section{- Figura 2. Opinión y práctica de abortos legales en hospitales públicos}

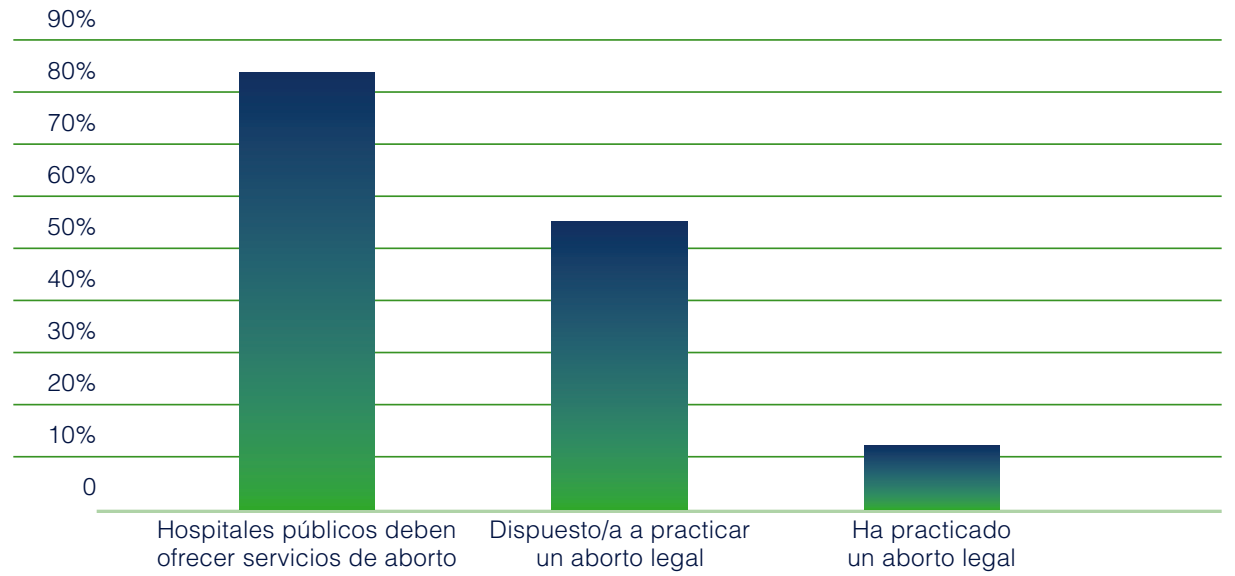

La discrepancia observada entre la actitud hacia el aborto y su práctica refleja la influencia de múltiples factores en la provisión de este servicio, tales como conocimientos médicos y legales, aspectos éticos, disponibilidad del equipo quirúrgico y personal médico y condiciones del hospital, entre otros. De hecho, en esta encuesta encontramos que los temas que despertaron mayor interés entre los médicos fueron los aspectos legales $(81 \%)$ y éticos $(65 \%)$ del aborto. 


\section{- Aspectos éticos}

El aborto es, en efecto, un tema ético. No existe una sola respuesta "correcta" sobre su práctica, ya que depende de los valores, creencias, deseos y circunstancias que rodean a cada persona. Se dice que existen dilemas éticos cuando "no hay una clara respuesta correcta o equivocada, sino que admiten argumentos a favor y en contra de cada postura." 49

La decisión sobre qué hacer ante un embarazo no deseado plantea un dilema ético, pues en éste se involucran valores sobre las concepciones que existen acerca de la vida humana y el debate sobre si el embrión debe ser considerado o no como una persona, lo mismo que acerca de la libertad y la responsabilidad de la mujer o la pareja involucrada en el embarazo.

El derecho a la libertad de conciencia protege la facultad humana de obrar conforme a los valores, creencias y pensamientos propios. El límite de este derecho, señalado por el artículo 18 del "Pacto Internacional sobre Derechos Civiles y Políticos" de Naciones Unidas es no vulnerar el derecho o la libertad de otra persona.

El derecho a la libertad de conciencia protege lo mismo a la mujer o la pareja que ha decidido interrumpir su embarazo que al prestador de servicios que lo realiza. El equilibrio entre la libertad personal y el deber del Estado de garantizar el ejercicio del derecho a la libertad de conciencia resulta en la protección de los derechos de la mujer y del prestador(a) de servicios, quien tiene la opción de declararse objeto de conciencia y abstenerse de practicar el aborto, refiriendo el caso a otro prestador(a); en el Distrito Federal específicamente, este equilibrio se expresa en los "Lineamientos generales de organización y operación de los servicios de salud relacionados con la interrupción del embarazo en el Distrito Federal". ${ }^{19}$ 
En el diálogo internacional, la interrupción del embarazo se incluye junto con otras circunstancias como una necesidad y derecho de la mujer, la adolescente y la pareja involucrada en un embarazo no deseado. La Conferencia Internacional de Población y Desarrollo de El Cairo (CIPD, 1994) y la Cuarta Conferencia Mundial de la Mujer realizada en Beijing (CCMM, 1995) mencionan el aborto como un problema de salud pública que es mayor en adolescentes de bajos recursos y que debe atenderse mejorando la calidad de los servicios públicos de salud sexual y reproductiva. De acuerdo con estos compromisos, los servicios de salud deben incluir la práctica del aborto legal en aquellos países que, como en México, el procedimiento es legal bajo ciertas circunstancias. La CIPD señala que los servicios de salud sexual y reproductiva deben incluir: el acceso a los servicios con calidad para la interrupción del embarazo, la atención de las complicaciones derivadas del aborto y también la consejería postaborto para evitar su repetición. ${ }^{50}$

El Comité para la Eliminación de Todas las Formas de Discriminación, ${ }^{51}$ que evalúa el seguimiento de los acuerdos de la Convención para la Eliminación de Todas las Formas de Discriminación contra la Mujer (México, 1998), obligatoria para el Estado Parte que lo ratifica, ha reconocido como derechos humanos aquéllos definidos por las Conferencias Internacionales en cuanto al derecho a la autodeterminación en la salud reproductiva y el acceso a los servicios de salud sexual y reproductiva, y ha recomendado al Estado Mexicano que todas las entidades del país puedan revisar su legislación y garantizar el acceso al aborto de manera rápida y ágil.

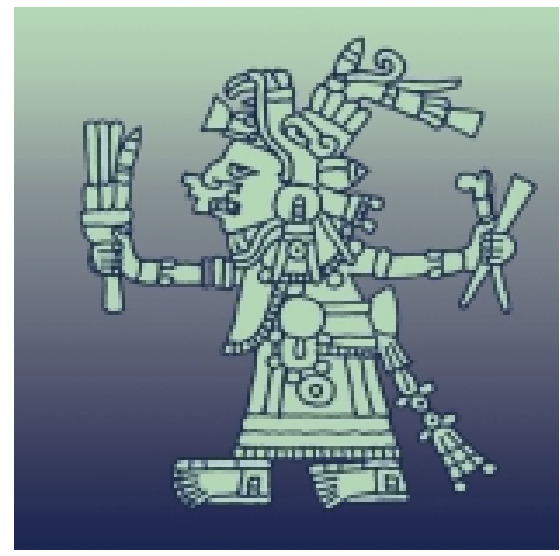

Chalchiuhtlicue, fuente de vida por excelencia. Fejérváry Mayer, 3.

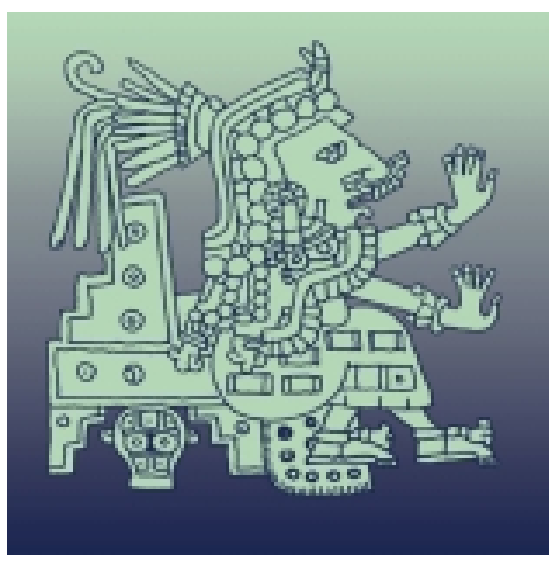

Chantico, arquetipo divino de la feminidad. Borgia, 63 


\section{- Consideraciones finales}

En los pasados cinco años el Gobierno del Distrito Federal, la Secretaría de Salud y organizaciones no gubernamentales han realizado una serie de acciones tendientes a aumentar el acceso de las mujeres al aborto legal en el D.F.

La modificación al Código Penal del D.F. realizada en el año 2000, permitió que se ampliaran las causales bajo las cuales el aborto es legal, que se definieran los procedimientos jurídicos para aprobar la realización de abortos legales por violación y que se obligara a los servicios de salud del D.F. a brindar estos servicios.

Como ya se dijo, la Secretaría de Salud del Distrito Federal, atendiendo estos cambios al Código Penal, emitió en el 2002 los "Lineamientos generales de organización y operación de los servicios de salud relacionados con la interrupción del embarazo en el Distrito Federal", en los cuales se establecen una serie de mecanismos, tiempos y condiciones bajo las cuales se deben brindar los servicios de aborto legal.

Posteriormente, la Secretaría de Salud del D.F., junto con organizaciones no gubernamentales dedicadas a la capacitación de prestadores de servicios de salud, y atendiendo los cambios en el Código Penal y las recomendaciones del Comité para la eliminación de todas las formas de discriminación contra la mujer (México, 1998), iniciaron un proceso de diagnóstico, información y sensibilización con 15 hospitales generales y materno-infantiles para entrenar a más de 500 médico(as), enfermeros(as) y trabajadores sociales, en aspectos legales, médicos y psicológicos de la interrupción legal del embarazo. Posteriormente se desarrolló un proceso de capacitación a grupos especializados de ocho hospitales que de acuerdo a los resultados del diagnóstico tenían posibilidades de realizar abortos legales.

Adicionalmente, existen tres hospitales de la Secretaría de Salud (SSA) que prestan servicios de aborto legal localizados en el D.F. y dos hospitales más en los estados de México e Hidalgo. Si bien estos servicios presentan algunas deficiencias en torno a la calidad de atención, cada vez se está ampliando más el acceso de las mujeres mexicanas a servicios seguros de aborto legal. 
Otro esfuerzo más por incrementar el acceso al aborto legal se inició recientemente (marzo, 2004) ${ }^{52}$ por parte de una organización no gubernamental (Grupo de Información en Reproducción Elegida, GIRE) en coordinación con la Procuraduría General de Justicia del D.F., la Secretaría de Salud local, el Instituto de las Mujeres del Distrito Federal, la Dirección de Salud Reproductiva de la UNAM y otras organizaciones no gubernamentales (Católicas por el Derecho a Decidir y Equidad de Género, Ciudadanía, Trabajo y Familia), al lanzar una amplia campaña de información para difundir que el aborto es legal en caso de violación y los procedimientos y requisitos necesarios para tener acceso a este servicio.

Las acciones realizadas en el D.F para aumentar el acceso a los servicios de aborto legal, así como el éxito en la coordinación de actividades entre el gobierno, la Procuraduría General de Justicia, la Secretaría de Salud y la sociedad civil, son experiencias positivas que deben promoverse en otros estados del país.

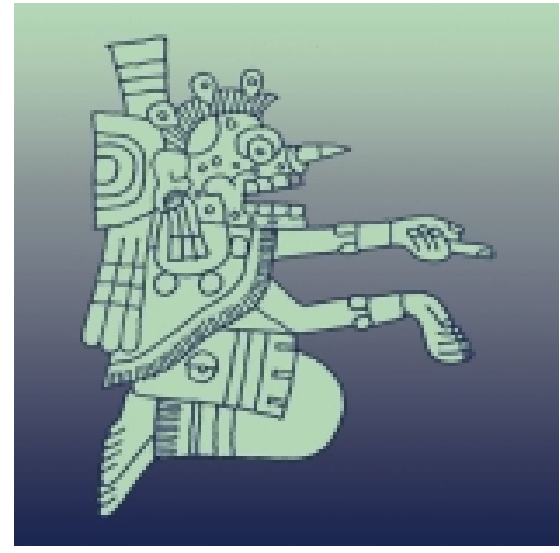

Mictlancihuatl, fuerza destructiva y creadora. Fejérváry Mayer, 37.

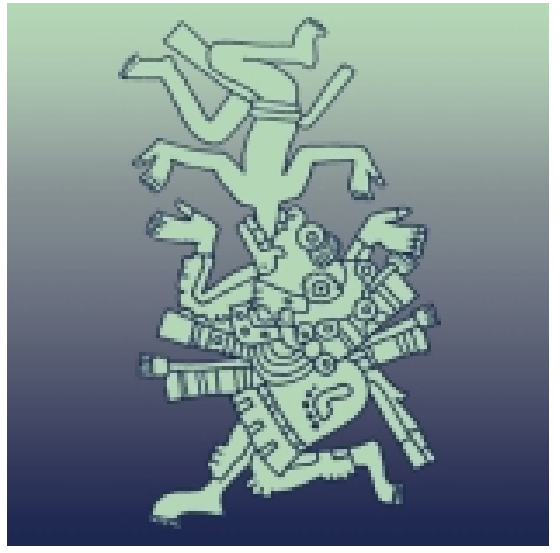

Mictlancihuatl, arquetipo de las mujeres que hacen ocurrir dentro de sí el milagro de la vida. Borgia, 5 


\section{Fuentes para obtener más información}

\section{INFORMACIÓN MÉDICA:}

- Abortion Facts dot Com

http://www.abortionfacts.com

- $\quad$ E-medical, get the facts online

http://www.emedical.com.au/default.asp?pagelD=5\&topic ID =88

- Ipas

http://www.ipas.org/english/womens_health/abortion/

- Misoprostol in Obstetrics and Gynaecology

http://www.misoprostol.org/miso_evidence.html

- $\quad$ Physicians for Reproductive Choice and Health - USA

http://www.prch

\section{ASPECTOS ÉTICOS Y LEGALES}

- GIRE

http://www.gire.org.mx/

- $\quad$ Católicas por el Derecho a Decidir

http://www.aciprensa.com/

- $\quad$ Center for Reproductive Law and Policy (CRLP)

http://www.reproductiverights.org

http://www.reproductiverights.org/pdf/sr_mex_1297_eng.pdf

- Comité de América Latina y el Caribe para la Defensa de los Derechos de la Mujer - Argentina www.cladem.org

- $\quad$ Catholics for a Free Choice - USA (inglés, español y portugués)

www.cath4chooice.org

- $\quad$ Pro Choice Forum (orientada a Inglaterra)

Sitio diseñado para interesados en aspectos sociales, legales y éticos del aborto http://www.prochoiceforum.org.uk

\section{ORGANIZACIONES, SOCIEDADES Y REDES}

- International Society of Abortion Doctors (ISAD), Holanda

El aborto: una guía práctica para médicos (inglés y español)

http://www.isad.org/prguide/p\%202\%20index.htm\#Index/contents

- $\quad$ Ibis Reproductive Health

http://www.ibisreproductivehealth.org/

- Medical Students for Choice (orientada a Estados Unidos y Canadá)

http://www.ms4c.org/

- National Abortion Federation-The Voice of Abortion Providers

http://www.prochoice.org/

- $\quad$ Doctors for Life

http://www.dfl.org.za/links/abortion_links.htm

- $\quad$ Planned Parenthood Association of America

http://www.plannedparenthood.org/abortion/default.htm

\section{BIBLIOTECAS Y FUENTES ADICIONALES}

- MedlinePlus

Un servicio de la Biblioteca Nacional de Medicina de Estados Unidos y de los Institutos Nacionales de Salud (NIH por sus siglas en inglés)

http://www.nlm.nih.gov/medlineplus/abortion.html

- Global Reproductive Health Forum (sitio muy interesante con vínculos a otros sitios)

http://www.hsph.harvard.edu/Organizations/healthnet/index.html

Específicamente sobre aborto: http://www.hsph.harvard.edu/Organizations/healthnet/abor/info.html

- Ipas

http://www.ipas.org/english/publications/abortion.html

- Instituto Alan Guttmacher

http://www.agi-usa.org/sections/abortion.html

- $\quad$ Early Options - USA

www.earlyoptions.org 


\section{Referencias}

1. Ahman E, y Shah I. Unsafe abortion: Worldwide estimates for 2000. Reproductive Health Matters 2002;10(19):13-17.

2. World Health Organization. Global and regional estimates of incidence of and mortality due to unsafe abortion, with a listing of available country data. WHO. Geneva, 1998.

3. Consejo Nacional de Población. Situación actual de la mujer en México. Diagnóstico sociodemográfico. Serie documentos técnicos. CONAPO. México, 2000.

4. Consejo Nacional de Población. Ejecución del Programa de Acción de la Conferencia Internacional sobre la Población y el Desarrollo. CONAPO. México, 1999.

5. The Alan Guttmacher Institute. Sharing responsibility: Women, society and abortion worldwide. AGI. New York, 1999.

6. The Alan Guttmacher Institute. Aborto clandestino: Una realidad latinoamericana. Nueva Cork: The Alan Guttmacher Institute;1994.

7. Finer, L.B. and K. Henshaw. Abortion Incidence and Services in the United States 2000. Perspectives on Sexual and Reproductive Health 2003;35(1):6-15.

8. Lamas M, Bissell S. Abortion and politics in Mexico: "Context is All". Reproductive Health Matters 2002;8(16):10-23.

9. Pick S, Givaduan M, Fawcett G. Resultado de tres encuestas nacionales de opinión sobre el aborto, México 1991-1993. Publicado en la memoria de la conferencia: Encuentro de Investigadores sobre Aborto Inducido en América Latina y el Caribe, Santa Fé Bogotá, 15-18 de noviembre, 1994. Universidad Externado de Colombia. Pp.85-103

10. Becker D, García S, Larsen Ulla. Knowledge and opinions about abortion law among Mexican youth International Family Planning Perspectives 2002;28(4):205-213.

11. García SG y Becker D. ¿Qué piensan y opinan las y los mexicanos sobre el aborto? Resultados de una encuesta nacional de opinión pública. Population Council y Grupo Investigación de Mercado y Asesoría, S.A. de C.V. México, 2001.

12. Saldaña A, Martínez M, Ortiz J, Catalán A. Conocimientos y actitudes de los profesionales de salud hacia el aborto inducido legal por causas genéticas. Presentado en el Congreso Nacional sobre Defectos al Nacimiento. México, 1999.

13. González de León D, Salinas A. Los médicos en formación y el aborto. Cad. Saúde Publ 1997;13(2):227-235.

14. González de León D, Salinas A. Resultados de una encuesta sobre aborto aplicada a residentes de la especialidad en ginecología y obstetricia en hospitales públicos de la ciudad de México. Reporte de investigación No. 89. Universidad Autónoma Metropolitana. México, 2000.

15. Casanueva E, Lisker R, Carnevale A, Alonso E. Attitudes of Mexican physicians toward induced abortion. International Journal of Gynecology and Obstetrics 1997;56: 47-52.

16. Torres E. Actitudes de los médicos tratantes de los abortos que se presentan en los hospitales del Distrito Federal. Population Council. México, 1993.

17. Feinholz-Klip D. El dilema del embarazo no previsto: visión del personal de salud. Rev. Perinatol Reprod Hum Méx 1999;13(1):112-125.

18. García S, Lara D y Goldman L. Conocimientos, actitudes y prácticas de los médicos mexicanos sobre el aborto: Resultados de una encuesta a nivel nacional. Gaceta Médica de México 2003; 139(1):S91-S102.

19. Gobierno del Distrito Federal, Secretaría de Salud del Distrito Federal. Lineamientos generales de organización y operación de los servicios de salud relacionados con la interrupción del embarazo en el Distrito Federal. Circular/GDF-SSD/02/02, En: Gaceta Oficial del Distrito Federal, México 2002;57:22-27.

20. Ladipo O. Preventing and managing complications of induced abortion in Third World countries. International Journal of Gynecology and Obstetrics 1989;(Suppl 3):21-8.

21. Kizza A, Rogo K. Assessment of the manual vacuum aspiration (MVA) equipment in the management of incomplete abortion. East African Medical Journal 1990 Nov;67(11):812-22

22. Baird T, Flinn S.. Manual vacuum aspiration: Expanding women's access to safe abortion services. Chapell Hill, NC., IPAS, 2001.

23. Koonin L, Strauss L, Chrisman C, Montalbano M, Bartlett L, Smith J. Abortion surveillance-United States, 1996. MMWR Morb Mortal Wkly Rep 1999;48(SS-4):1-42.

24. Huntington D, Nawar L, Hassan E, Youssef H, Abdel-Tawab N. The postabortion caseload in Egyptian hospitals: A descriptive study. International Family Planning Perspectives 1998; 24(1):25-31

25. Westfall J, Sophocles A, Burggraf H, Ellis S. Manual vacuum aspiration for first-trimester abortion. Archives in Family Medicine 1998;7:559-662. 
26. Fuentes J, Billings D. A comparison of three models of postabortion care in Mexico. Final report. Population Council. New York, 1998.

27. Johnson B, Benson J, Bradley J, and Ordoñez A. Cost and resource utilization for the treatment of incomplete abortion in Kenya and Mexico. Social Science and Medicine 1993; 36(11):1443-1453.

28. Brambila C, Langer A, García C, Molina A, Heimburger A, Barahona V. Análisis de costos de los servicios postaborto en el Hospital General Dr. Aurelio Valdivieso, Oaxaca, México. Population Council, 1998.

29. Staley D, Maltzer M, Wiebe E, Halvorson-Boyd G, Boyd C. Pain Management in: Maureen P, Lichtenberg S, Boratta L, Grimes D. Stubblefield P. A clinician's guide to medical and surgical abortion. Churchill Livingstone New York, 1999. Pp. 73-89.

30. Castleman L, Mann C. Manual vacuum aspiration (MVA) for uterine evacuation: Pain management. Chapel Hill, NC, Ipas.

31. World Health Organization (WHO). Complications of abortion: Technical and managerial guidelines for prevention and treatment, Geneva: WHO 1995.

32. World Health Organization (WHO). Managing complications in pregnancy and childbirth (MCPC): A guide for midwives and doctors. WHO. 2002. http://www.who.int/reproductive-health/impac/index.html

33. González de León D, Billings D, Gasman N, Ramírez R. Incorporación de la salud sexual y reproductiva y del enfoque de la atención integral a la mujer con aborto en los programas de licenciatura de medicina. IPAS. México, 2002.

34. Quiróz G, Billings D, Gasman N. Aspiración manual endouterina (AMEU): Tecnología adecuada para la atención de calidad a mujeres en situación de aborto. Gaceta Médica de México 2003; 139(Suplemento No.1):S65-S73

35. Stewart F, Wells E, Flinn S, Weitz T. Aborto temprano con asistencia médica: temas para la práctica. UCSF Center for Reproductive Health Research \& Policy, San Francisco, California 2001.

36. Spitz IM, Bardin CW. Clinical pharmacology of RU. 486, an antiprogestin and antiglucocorticoid. Conctraception 1993; 48:403-44.

37. Espinoza H, Norris A, Díaz-Olavarrieta C. Carpeta educativa: Medicamentos para la interrupción legal del embarazo temprano, aborto medicamentoso. México, D.F. México: Population Council, 2003.

38. Clark S, Blum J, Blanchard K, Galvao L, Fletcher H, Winikoff B. Misoprostol use in obstetrics and gynecology in Brazil, Jamaica and the United States. International Journal of Gynecology and Obstetrics 2002; 76:65-74

39. Blanchard K, Winikoff B, Ellertson C. Misoprostol used alone for the termination of early pregnancy. A review of evidence. Contraception 1999:59:209-217.

40. Pymar HC, Creinin MD. Alternatives to mifepristone regimens for medical abortion. American Journal of Obstetrics and Gynecology 2000; 183:S54-S64.

41. Goldberg AB, Geenberg MB, Darney PD. Misoprostol and pregnancy. New England Journal of Medicine 2001; 344:38-47.

42. Creinin MD, Vittinghoff E, Galbraith S, Klaisle C. A randomized controlled trial comparing misoprostol three and seven days following methotrexate for early abortion. American Journal of Obstetrics and Gynecology 1995;173:1578-84.

43. Lipscomb G, Stovall T, Ling G. Nonsurgical treatment of ectopic pregnancy. New England Journal of Medicine 2000; 343: 1325-1329.

44. Carbonell J, Varela L, Velazco A, Fernández C, Sánchez C. The use of misoprostol for abortion at < or $=9$ weeks' gestation. Eur J Contracept Reprod Helath Care. 1997a; 2(3):181-5.

45. Carbonel J, Varela L, Velazco A, Fernández C. The use of misoprostol for termination of early pregnancy. Contraception 1997b; 55(3):165-8.

46. Jain J, Mekstroth K, Mishell D. Early pregnancy termination with intravaginal administered sodium chloride solution-moistened misoprosol tablets: Historial comparison with mifepristone and oral misprosto. Am J Ostet Gynecol 1999:59:219-225.

47. Tang O, Xu J, Cheng L, Lee SW, Ho PC. Pilot sutyd on the use of sublingual misoprostol with mifepristone in the termination of firt trimester pregnancy up to 9 weeks gestation. Human Reproduction 2002;17(7):1738-40.

48. Pang M, Lee T, Chung T. Incomplete miscarriage: a randomized controlled trial comparing oral with vaginal misoprostol for medical evacuation. Human Reproduction 2001; 16(11):2283-2287.

49. Macklin Ruth. Dilemas. Los problemas éticos y morales. Atlántida, Buenos Aires, 1995

50. Organización de las Naciones Unidas. Informe de la Conferencia Internacional sobre la Población y el Desarrollo. UN Population Division, Department of Economic and Social Affairs, Population Fund. Egipto. El Cairo, 18 de octubre de 1994. UN Doc. No. A/CONF.171/13; 8.19

51. Office of the High Commissioner for Human Rights, La mujer y la salud:.02/02/99 CEDAW Recom. general 24. General comments. 20o periodo de sesiones, Geneva, Switzerland, 1999.

52. Grupo de Información en Reproducción Elegida (GIRE). Campaña para que las mujeres puedan ejercer su derecho a un aborto legal. Boletín de prensa. México: 2 de Marzo 2003. 